\title{
Glomus Tumor of the Hand
}

\author{
Won Lee ${ }^{1}$, Soon Beom Kwon ${ }^{1}$, Sang Hun $\mathrm{Cho}^{2}$, Su Rak Eo ${ }^{2}$, Chan Kwon ${ }^{2}$ \\ ${ }^{1}$ Yonsei E1 Plastic Surgery, Anyang; ${ }^{2}$ Department of Plastic and Reconstructive Surgery, Dongguk University Medical Center, Dongguk \\ University Graduate School of Medicine, Goyang, Korea
}

Background Glomus tumors were first described by Wood in 1812 as painful subcutaneous tubercles. It is an uncommon benign neoplasm involving the glomus body, an apparatus that involves in thermoregulation of cutaneous microvasculature. Glomus tumor constitutes 1\%$5 \%$ of all hand tumors. It usually occurs at the subungual region and more commonly in aged women. Its classical clinical triad consists of pain, tenderness and temperature intolerance, especially cold sensitivity. This study reviews 15 cases of glomus tumor which were analyzed according to its anatomic location, surgical approach and histologic findings.

Methods Fifteen patients with subungual glomus tumors of the hand operated on between January 2006 and March 2013, were retrospectively reviewed. Patients were evaluated preoperatively with standard physical examination including ice cube test and Love's test. Diagnostic imaging consisted of ultrasonography, computed tomography, and magnetic resonance imaging. All procedures were performed with tourniquet control under local anesthesia. Eleven patients underwent excision using the transungual approach, 3 patients using the volar approach and 1 patient using the lateral subperiosteal approach.

Results Total of 15 cases were reviewed. 11 tumors were located in the nail bed, 3 in the volar pulp and 1 in the radial aspect of the finger tip. After complete excision, patients remained asymptomatic in the immediate postoperative period. In the long term follow up, patients exhibited excellent cosmetic results with no recurrence.

Conclusions Accurate diagnosis should be made by physical, radiologic and pathologic examinations. Preoperative localization and complete extirpation is essential in preventing recurrence and subsequent nail deformity.

Keywords Glomus tumor / Neoplasms / Hand
Correspondence: Sang Hun Cho Department of Plastic and Reconstructive Surgery, Dongguk University Medical Center, Dongguk University Graduate School of Medicine, 27 Dongguk-ro, llsandonggu, Goyang 410-773, Korea Tel: +82-31-961-7330

Fax: +82-31-961-7347

E-mail: chosh@dumc.or.kr
No potential conflict of interest relevant to this article was reported.

Received: 21 Jul 2014 • Revised: 31 Oct 2014 • Accepted: 31 Oct 2014

pISSN: 2234-6163 • elSSN: 2234-6171 • http://dx.doi.org/10.5999/aps.2015.42.3.295 • Arch Plast Surg 2015;42:295-301

\section{INTRODUCTION}

Glomus tumors occur relatively rarely, accounting for about $1 \%-5 \%$ of all hand tumors. Although they can develop in any part of the body, they commonly do so in the upper extremities-most frequently subungual areas. They are characterized by stabbing paroxysmal pain in the fingertips, tenderness, and cold intolerance $[1,2]$.
A glomus body is an apparatus of the skin and a type of an arteriovenous anastomosis that controls the body temperature. Glomus tumors refer to hyperplasia of the glomus body. Their pathological findings were first described by Masson [3] in 1924, being referred to as tumors that occur in the neuromyoarterial body.

Glomus tumors are categorized into solitary and multiple types according to their clinical presentations. Solitary glomus 
tumors accompany pain and occur mainly in the distal extremities; multiple glomus tumors are usually painless and can develop in any part of the body [4]. Glomus tumors require careful assessment for differential diagnosis, because they can often be misdiagnosed and followed by unsuitable treatment, due to the chronic pain in all extremities they accompany [5].

The authors of the study reviewed 15 hand glomus tumor cases that they experienced in the previous eight years and analyzed the data by patient age, sex distribution, location of the tumors, chief complaint, preoperative tests, surgical treatment, and biopsy results. The results of the study are presented here along with a literature review.

\section{METHODS}

The subjects of this study were 15 hand glomus tumor patients whose diagnosis was confirmed with biopsy results from Janu-

\section{Fig. 1. Image of color Doppler ultrasonography}

Color Doppler ultrasonographic image shows a tiny ovoid, hypervascular nodule beneath the nail, with focal bone erosion of the distal phalanx (white arrow).

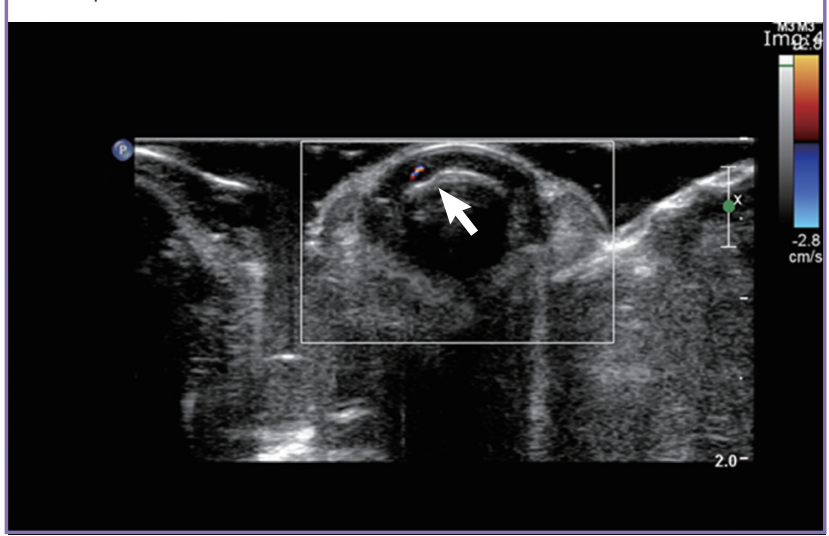

Fig. 2. Axial gadolinium-enhanced fat-saturated T1weighted image

Axial gadolinium-enhanced fat-saturated T1-weighted image shows a prominent enhancing nodule with subtle erosion at the distal phalanx (white arrow).

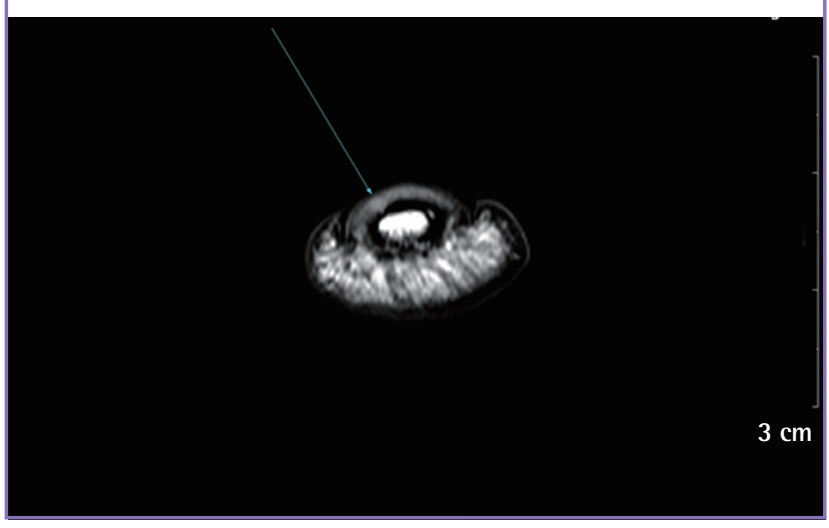

ary 2006 to March 2013. All of them were admitted with a chief complaint of pain in a subcutaneous nodule in the hand. In all cases, the pain was caused by pressure, but in one case, the pain occurred as cold intolerance. One case was recurrent after undergoing surgical treatment in other hospital. Three cases demonstrated nail deformities. Seven exhibited bluish discoloration in the nails. All of the patients had a solitary glomus tumor in one digit.

For diagnosis, all of the patients underwent a simple ice cube test and simple X-ray test. Three patients showed abnormal findings including bone erosion. Ultrasonography (Fig. 1), computed tomography (CT), and magnetic resonance imaging (MRI) (Fig. 2) were additionally performed in cases where no pain was induced by the ice cube test or no abnormal findings were discovered in the simple X-ray test.

Operations were performed under a digital nerve block with $2.5 \times$ magnifying loupes, and a tourniquet was applied. The specific location of the tumor was outlined again before the anesthesia because the position might be confused after anesthesia. A typical transungual approach was employed in 11 cases. In three cases where tumors were located in the volar area, an incision line was drawn on the tumors, and the tumors were excised. A lateral subperiosteal approach - in which an incision line was drawn in the lower lateral side of the nail fold and the nail bed was raised to extract the tumor-was employed in one case.

In performing operations using the transungual approach, a periosteal elevator was inserted between the fingernail and nail bed. The nail was avulsed, with care not to damage the nail bed. Then the fingernail was raised from the bed and nail fold, exposing the nail bed where the tumor was present. A relatively welldefined tumor was seen. The nail bed was incised longitudinally, and the tumor was carefully detached and removed completely (Fig. 3). The primary suture was made using Vicryl 7-0 absorbable suture, and the nail plate was repositioned after being closed with nylon 4-0.

Fig. 3. Various methods of surgical approach

(A) Transungal approach, (B) lateral subperiosteal approach, (C) volar approach.
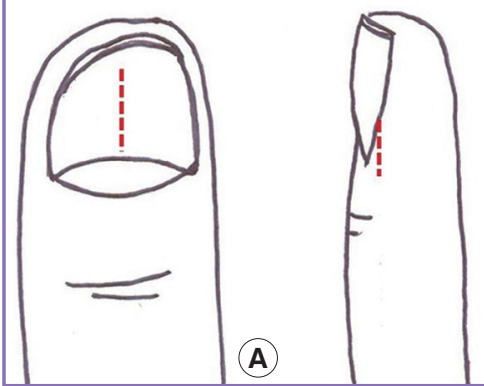

(B)

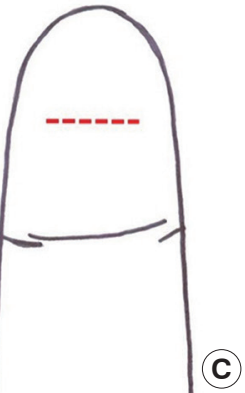




\section{RESULTS}

The tumors that were removed were in an ovoid or round shape, 3-9 $\mathrm{mm}$ in size. In 13 cases, they were covered in a capsule and thus were removed clearly from the surrounding tissues (Fig. 4). In contrast, in one case where the tumor was a glomangioma (Fig. 5), no capsule was observed. The remaining one case was a glomangiomyoma (Table 1, Fig. 6).

In all of the patients, the pain either disappeared or was alleviated; no recurrence or complications including postoperative nail deformity were seen during the follow-up period.

Histopathologically, the tumors were covered in relatively well-defined fibrous capsules in a low-power field. Epitheloid cells with regular shapes surrounded the dilated and thin-walled blood vessels in a single or multiple layers. In a high-power field, epitheloid cells with an ovoid or round nucleus that had almost no mitotic figures were observed.

\section{DISCUSSION}

Glomus tumors occur as some tissues of a glomus body prolifer- ate. A glomus body is a specialized arteriovenous anastomosis responsible for thermoregulation and is located in the reticular layer of the dermis. Glomus tumors arise from deformed smooth muscle cells called glomus cells, which constitute a normal glomus body and are located in the walls of the SucquetHoyer canal. These cells are present throughout the entire body. They mostly develop in the skin, but are also reported to arise in the mucous membrane or internal organs such as the stomach, lung, trachea, and bone $[4,6,7]$. The most common site for glomus tumors is the hand-particularly the subungual area, the lateral aspect of the digits, and the palms [8].

Glomus tumors are categorized as either solitary or multiple, according to their clinical presentation. Solitary glomus tumors are far more common than the multiple variant. According to Rettig and Strickland [9], the solitary variant is mostly found in the hand, with $25 \%-75 \%$ occurring in the subungual region, which are more frequent in women. Multiple glomus tumors are reportedly painless in general, of a pink or purple nodal shape, and develop in young children or males in an autosomal dominant fashion $[2,10]$.

Among the accompanying symptoms, paroxysmal pain, tender-

Fig. 4. Gross and pathologic feature of glomus tumor

(A) Gross appearance. (B) Tumor resection with a transungual approach. Pathologic features of a glomus tumor. (C) The tumor cell has a sharply punched-out rounded nucleus with amphophilic or pale eosinophilic cytoplasm $\left(H \& E_{1} \times 400\right)$. (D) The lacework of basement membrane around the tumor cells is accentuated with a periodic acid-Schiff stain (Periodic acidSchiff stain, $\times 400$ ).

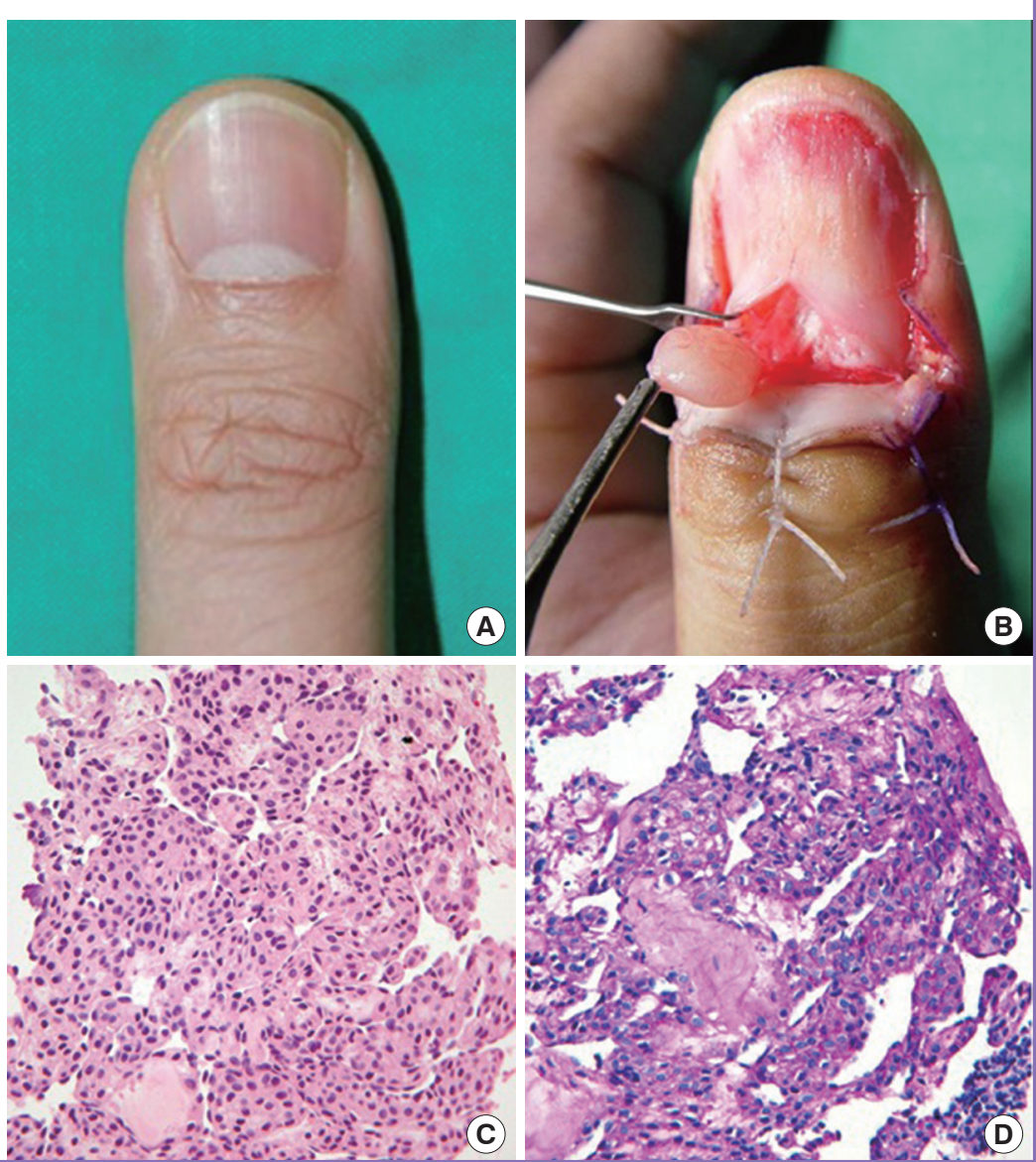




\section{Fig. 5. Gross and pathologic feature of glomangioma}

(A) Gross appearance. (B) Tumor resection with careful preservation of the nail bed. (C) Pathologic features of glomangioma. The tumor has hemangiomalike multiple dilated blood vessels with surrounding clusters of glomus cells $\left(H \& E_{1} \times 400\right)$. (D) The dilated blood vessels are conspicuous in the immunohistochemical stain for CD34. The tumor cells show membranous reactivity for CD34 (Immunohistochemistry for $\mathrm{CD} 34, \times 400)$.
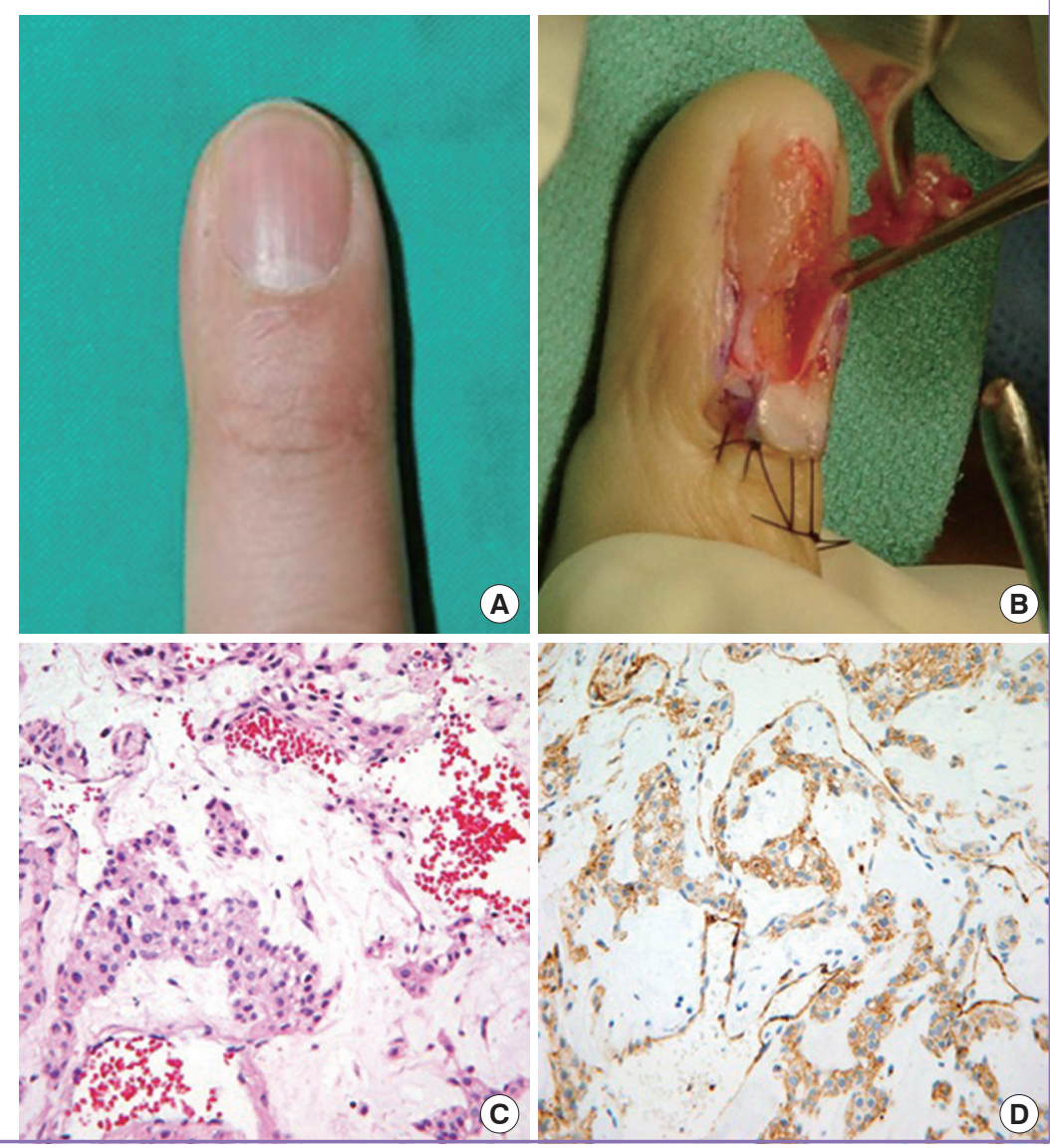

Table 1. Patient details

\begin{tabular}{|c|c|c|c|c|c|c|c|c|}
\hline Case & $\begin{array}{l}\text { Age } \\
(\mathrm{yr})\end{array}$ & Sex & Location & $\begin{array}{l}\text { Size } \\
(\mathrm{mm})\end{array}$ & Symptoms & $\begin{array}{l}\text { Imaging } \\
\text { study }\end{array}$ & Method & Result \\
\hline 1 & 52 & Female & Left thumb, subungal & 3 & Pain, tenderness, dislocation & MRI & Transungual & Glomus tumor \\
\hline 2 & 35 & Male & Left 5th finger, subungal & 9 & Pain, tenderness & X-ray, US, MRI & Transungual & Glomangioma \\
\hline 3 & 47 & Female & Right 3rd finger, subungal & 4 & Pain, tenderness & X-ray, MRI & Transungual & Glomus tumor \\
\hline 4 & 44 & Male & Right 2nd finger, subungal & 6 & $\begin{array}{l}\text { Pain, tenderness, dislocation, } \\
\text { nail deformity }\end{array}$ & X-ray, US & Transungual & Glomus tumor \\
\hline 5 & 30 & Female & Right 5th finger, subungal & 5 & Pain, tenderness & X-ray, US & Volar & Glomus tumor \\
\hline 6 & 34 & Female & Left 4th finger, subungal & 4 & Pain, tenderness, dislocation & US & Transungual & Glomus tumor \\
\hline 7 & 49 & Female & Left 4th finger, subungal & 5 & $\begin{array}{l}\text { Pain, tenderness, dislocation, } \\
\text { nail deformity }\end{array}$ & X-ray, US & Transungual & Glomus tumor \\
\hline 8 & 38 & Female & Right 3rd finger, volar & 5 & Pain, tenderness & X-ray, US & Volar & Glomus tumor \\
\hline 9 & 52 & Female & Left thumb, volar & 8 & Pain, tenderness & X-ray, US & Volar & Glomangiomyoma \\
\hline 10 & 36 & Female & Right 3rd finger, subungal & 3 & Pain, tenderness, cold sensitivity & X-ray, US & Transungual & Glomus tumor \\
\hline 11 & 32 & Female & Left thumb, subungal & 4 & Pain, tenderness & X-ray, US, MRI & Transungual & Glomus tumor \\
\hline 12 & 37 & Male & Left thumb, subungal & 6 & Pain, tenderness & US & Transungual & Glomus tumor \\
\hline 13 & 35 & Female & Left 5th finger, subungal (recurred) & 4 & Pain, tenderness, dislocation & X-ray, US, MRI & Transungual & Glomus tumor \\
\hline 14 & 76 & Female & Right 4th finger, radial aspect & 8 & $\begin{array}{l}\text { Pain, tenderness, dislocation, } \\
\text { nail deformity }\end{array}$ & X-ray, US & Lateral subperiosteal & Glomus tumor \\
\hline 15 & 41 & Male & Right 5th finger, subungal & 5 & Pain, tenderness, dislocation & X-ray, US & Transungual & Glomus tumor \\
\hline
\end{tabular}

ness, and aggravation of the symptoms by coldness are significant in reaching diagnosis. Other symptoms include the distinctive subungual discoloration, hypoesthesia, atrophy, osteoporosis in the lesion, and autonomic disturbance such as Horner syndrome.

Various hypotheses are suggested regarding the cause of pain in glomus tumors: the capsules of the tumors render them sen- 
Fig. 6. Images showing pathologic features of glomangiomyoma

(A) The tumor is composed of proliferating spindle smooth muscle cells with dilated blood vessels as well as round glomus cells. Characteristically, the glomus cells undergo a transition to spindle smooth muscle cells $\left(H \& E_{1} \times 400\right)$. (B) The spindle smooth muscle cells and the round glomus cells are both positive for smooth muscle actin (immunohistochemistry for smooth muscle actin, $\times 400$ ).

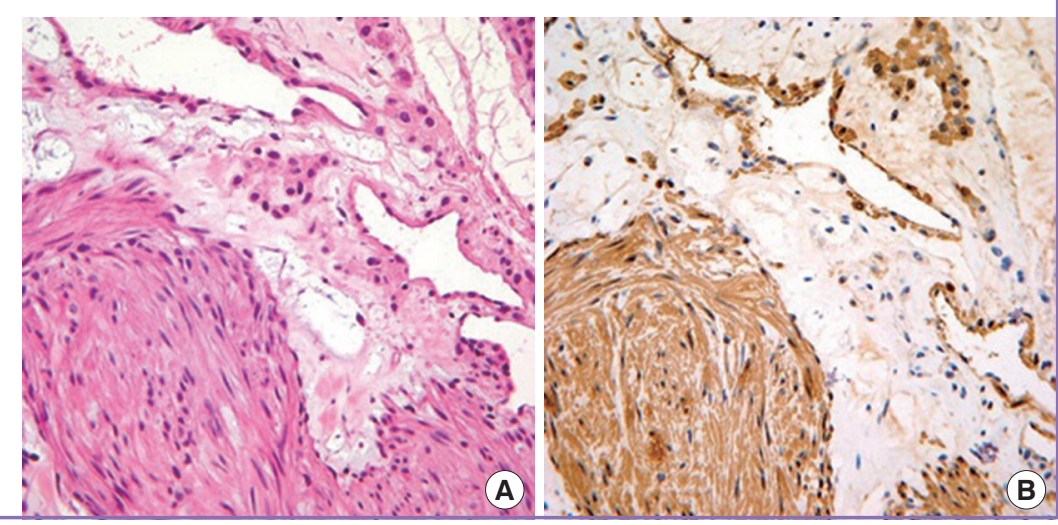

sitive to pressure; abundant mast cells in the glomus tumors release substances such as heparin, 5-hydroxytryptamin, and histamine, causing receptors to pressure or cold stimulation to be sensitive. Such factors incite severe pain even to ordinary stimulation [1]. Furthermore, excessive dominance over the nerve of numerous non-myelinated nerve fibers that penetrate into glomus tumors has been identified as a cause of pain [11].

If glomus tumors occur in the subungual area of the digits, diagnosis can be made solely based on an accurate health history intake and physical examinations. However, in many cases, such clinical symptoms are not clear-cut, and lesions are difficult to locate, as they are usually smaller than $1 \mathrm{~cm}$. For such reasons, clinicians need to check whether the surrounding tissues of the lesions become pale when the nail is pressured, even when no discoloration under the nail is noted. Clinical diagnostic tests including Love's pin test, Hildreth's test, or a cold-sensitivity test can be helpful, but still have limitations in leading to accurate diagnosis $[5,6,8]$. Positive Love's pin test means patient experience severe pain and reduction in pain when the skin overlying the tumor is pressed with a pinhead, ballpoint pen, end of a paperclip, or Kirschner wire. Hildreth's test is performed by elevating the patients' arm to exsanguinate it. A tourniquet is inflated to $250 \mathrm{~mm}$ $\mathrm{Hg}$ and the tumor is palpated, the pain and tenderness should be reduced. A test is positive when releasing the cuff causes a sudden onset of pain and tenderness in the area of the tumor. The coldsensitivity test is positive when immersing the hand in cold water elicits severe pain in and around the lesion [12].

Solitary glomus tumors need to be ruled out from painful tumors, such as leiomyoma or eccrine spiradenoma. As in many cases, a diagnosis can easily be delayed for a long time while the patient suffers from severe pain because patients are easily misdiagnosed with conditions like neuropathic complaints, arthritis, or neuralgia. Therefore, they may undergo unsuitable treatment including sympathetic ganglionectomy, radicotomy, or even dismemberment $[1,13]$. Clinicians need to be particularly careful to rule out cavernous hemangioma and blue rubber-bleb nevus syndrome from multiple glomus tumors, as they can be easily confused with one another $[4,14]$.

Additional tests such as a simple X-ray, CT, angiography, and ultrasonography can be conducted for more accurate diagnosis. MRI can also be useful for cases with less distinct clinical signs and symptoms. When performing a simple X-ray test, inciting pressure atrophy to a distal phalanx can yield findings that seem like osteocystoma or erosion, or no abnormal findings. Thus, diagnosis can be made based on the unique simple $\mathrm{X}$-ray test findings after giving pressure to a distal phalanx if more than two years have passed since the occurrence of a tumor. However, diagnosing early stage lesions requires more careful physical examinations and additional imaging tests.

With regard to diagnosing glomus tumors using ultrasonography, Fornage [15] reported that he was able to localize the lesions in 75 percent of cases using a $7.5 \mathrm{MHz}$ probe. However, he recommended the use of a $10 \mathrm{MHz}$ probe, as a $5.0 \mathrm{MHz}$ probe cannot easily detect small and flat tumors. MRI is not invasive and it provides excellent contrast between a neoplasm and normal tissue. Glomus tumors are described as slightly hypointensive to slightly hyperintensive on a T1-weighted image, and hyperintensive on a T2-weighted image. The T1-weighted image after injection of gadolinium shows a more strong enhancement, and the lesion can be seen more clearly. It is essential to highlight disruptions of the capsule and areas of enhancement beside the main tumor $[16,17]$. Ultrasonography can be a better option than MRI, considering the time required for the test, its cost, and its ability to enable the evaluation of lesions dynamically in real-time [18].

In treating glomus tumors, a complete surgical excision is known to be the best way to relieve pain and prevent recurrence $[1,2,5,6,8]$. In performing operations, removal in a bloodless field with tourniquet application is necessary. Total extirpation is performed after carefully avulsing the surrounding tissues of 
the tumors. In cases where the operation field is not clear due to hemorrhage, total extirpation of the tumors is often impossible and the recurrence of tumors is highly likely [13]. If the tumors have penetrated into the bones, bone curettage or partial excision is performed. Unless there is a malignant finding, en bloc resection is not necessary [19].

Among the various surgical approaches that have been devised for complete excision, a common choice for tumors located under the nails is a transungual approach. In this method, the nails are removed, and an excision is made on the ventral surface of the nail bed where the tumors are present in order to remove the tumors. Although this approach is advantageous in offering a good field of view, it can yield cosmetically unsatisfying results if the nail bed is severely damaged while removing tumors or if sutures of the nail bed are made without care, causing postoperative nail deformity.

In an attempt to avoid such nail deformity, an approach to removing tumors by elevating a flap under the periosteum through a lateral incision of the nail bed has been introduced [5]. However, application of the method can be limited by the location of tumors, and the procedure is complicated. Moreover, the technique can sometimes damage digital nerves. Another approach to preventing a nail deformity has been suggested: A "U"-shaped incision is made to the upper nail where a tumor is located. Then, a Greenstick fracture is made on the nail bed to partially open it in a trapdoor shape. That way the nail bed where the tumor exists is exposed [20]. Through such an approach, the nail bed is maintained flat until new nails grow and nail deformities can be prevented [21].

Histopathologically, glomus tumors consist of glomus cells, blood vessels, and smooth muscles. Glomus tumors are categorized into three types by the composition of these components: glomangiomas are mainly made up of the vessels; solid glomus tumors are chiefly composed of glomus cells; and glomangiomyomas show a predominance of smooth muscles. Although glomus tumors are essentially benign, sarcomas accompany benign glomus tumors in rare cases to form glomangiosarcoma [4].

The postoperative recurrence of tumors in a localized area is usually caused by the incomplete removal of the tumors, but may also signify multiple glomus tumors. Therefore, meticulous care needs to be given at the first operation to completely remove all lesions. To prevent incomplete excision, a good view needs to be ensured in removal as well. Performing curettage additionally in order to remove remaining tissues after the excision is also useful.

In cases where patients with solitary or multiple subcutaneous nodules in the extremities - particularly in the hand — are admitted with chronic pain, clinicians need to provide thorough physical assessment and preoperative tests to rule out glomus tumors. Once a differential diagnosis is made, complete surgical excision of the lesions needs to be done. Despite the low occurrence of multiple glomus tumors, clinicians should also keep the possibility of the tumors in mind and perform careful examinations and preoperative tests.

\section{REFERENCES}

1. Lee $\mathrm{CH}$, Byeon JH, Rhie JW, et al. Clinical analysis of twenty cases of glomus tumor in the digits. J Korean Soc Plast Reconstr Surg 1995;22:169-78.

2. Carroll RE, Berman AT. Glomus tumors of the hand: review of the literature and report on twenty-eight cases. J Bone Joint Surg Am 1972;54:691-703.

3. Masson P. Le glomus neuro-myo-arteriel des regions tactiles et ses tumeurs. Lyon Chi 1924;21:257-80.

4. Chatterjee JS, Youssef AH, Brown RM, et al. Congenital nodular multiple glomangioma: a case report. J Clin Pathol 2005;58:102-3.

5. Vasisht B, Watson HK, Joseph E, et al. Digital glomus tumors: a 29-year experience with a lateral subperiosteal approach. Plast Reconstr Surg 2004;114:1486-9.

6. Rohrich RJ, Hochstein LM, Millwee RH. Subungual glomus tumors: an algorithmic approach. Ann Plast Surg 1994; 33:300-4.

7. Shugart RR, Soule EH, Johnson EW Jr. Glomus tumor. Surg Gynecol Obstet 1963;117:334-40.

8. Van Geertruyden J, Lorea P, Goldschmidt D, et al. Glomus tumours of the hand: a retrospective study of 51 cases. J Hand Surg Br 1996;21:257-60.

9. Rettig AC, Strickland JW. Glomus tumor of the digits. J Hand Surg Am 1977;2:261-5.

10. Beasley SW, Mel J, Chow CW, et al. Hereditary multiple glomus tumours. Arch Dis Child 1986;61:801-2.

11. Rodriguez JM, Idoate MA, Pardo-Mindan FJ. The role of mast cells in glomus tumours: report of a case of an intramuscular glomus tumour with a prominent mastocytic component. Histopathology 2003;42:307-8.

12. Hazani R, Houle JM, Kasdan ML, et al. Glomus tumors of the hand. Eplasty 2008;8:e48.

13. Takei TR, NalebuffEA. Extradigital glomus tumour.J Hand Surg Br 1995;20:409-12.

14. Greider JL Jr, Flatt AE. Glomus tumor associated with pacinian hyperplasia: case. J Hand Surg Am 1982;7:113-7.

15. Fornage BD. Glomus tumors in the fingers: diagnosis with US. Radiology 1988;167:183-5.

16. Drape JL. Imaging of tumors of the nail unit. Clin Podiatr 
Med Surg 2004;21:493-511.

17. Goettmann S, Drape JL, Idy-Peretti I, et al. Magnetic resonance imaging: a new tool in the diagnosis of tumours of the nail apparatus. Br J Dermatol 1994;130:701-10.

18. Moon ES, Choi MS, Kim MS, et al. Distribution of glomus tumors in fingers. J Korean Soc Surg Hand 2009; 14:138-43.

19. Kim JH, Yim HW, Yoon CS, et al. Glomus tumor in subcutaneous layerof forearm: a case report. J Korean Soc Surg
Hand 2009; 14:92-4.

20. Kwon ST, Jeong EC. Subungual glomus tumor resection with partial detachment of nail plate. J Korean Soc Surg Hand 2009; 14:210-4.

21. Tada H, Hirayma T, Takemitsu Y. Prevention of postoperative nail deformity after subungual glomus resection.J Hand Surg Am 1994;19:500-3. 